\title{
Aberrant DJ-1 expression underlies L-type calcium channel hypoactivity in tuberous sclerosis complex and Alzheimer's disease
}

Farr Niere ${ }^{1}$, Luisa P. Cacheaux ${ }^{1}$, Ayse Uneri ${ }^{1}$, Sanjeev Namjoshi ${ }^{1}$, Cameron

Reynoldson ${ }^{1}$, Juan Penaranda ${ }^{1}$, William C. Taylor ${ }^{1}$, Suzanne Craft ${ }^{2}$, Christopher Dirk Keene ${ }^{3}$, Tao $\mathrm{Ma}^{1,2}$, and Kimberly F. Raab-Graham ${ }^{1 *}$

Affiliations: ${ }^{1}$ Department of Physiology and Pharmacology, ${ }^{2}$ Department of Gerontology and Geriatric Medicine, Wake Forest School of Medicine, Medical Center Boulevard, Winston-Salem, NC 27157, USA; ${ }^{3}$ Department of Pathology, University of Washington School of Medicine, Seattle, WA 98104, USA.

* To whom correspondence should be addressed: Kimberly F. Raab-Graham, Ph.D. Department of Physiology and Pharmacology, Wake Forest School of Medicine, Medical Center Boulevard, Winston-Salem, NC 27157-1083

E-mail: kraabgra@wakehealth.edu 


\section{Abstract}

L-type voltage-dependent $\mathrm{Ca}^{2+}$ channels (L-VDCC) integrate synaptic signals to facilitate a plethora of cellular mechanisms. L-VDCC dysfunction is implicated in several neurological and psychiatric diseases. Despite their importance, signals upstream of LVDCC activity that regulate their channel density, however, are poorly defined. In disease models with overactive mammalian target of rapamycin complex 1 (mTORC1) signaling (or mTORopathies), including tuberous sclerosis (TS) and Alzheimer's disease (AD), we report a novel mechanism downstream of mTORC1 signaling that results in a deficit in dendritic L-VDCC activity. Deficits in L-VDCC activity are associated with increased expression of the mTORC1-regulated RNA-binding protein DJ-1. DJ-1 binds the mRNA coding the auxiliary $\mathrm{Ca}^{2+}$ channel subunit $\alpha 2 \delta 2$ responsible for shuttling LVDCC to the membrane and represses its expression. Moreover, this novel DJ1/a282/L-VDCC pathway is disrupted in human AD and preclinical models of $A D$ and TS. Our discovery that DJ-1 directs L-VDCC activity and L-VDCC-associated protein

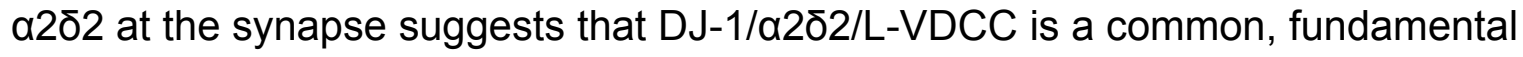
pathway disrupted in TS and AD that can be targeted in clinical mTORopathies.

\section{Keywords}

alpha2delta2 ( $\alpha 2 \delta 2)$; Alzheimer's disease; autism spectrum disorder; Cacna2d2, calcium channels; disease-disease interaction; DJ-1; epilepsy; mammalian target of rapamycin (mTOR); mRNA; RNA-binding protein; Parkinsonism associated deglycase; 
Parkinson disease (autosomal recessive, early onset) 7; synapses; tuberous sclerosis complex

\section{Significance Statement}

Many neurological disorders share symptoms, despite disparity among diseases.

Treatments are prescribed based on diagnosis rather than individual symptoms. While only treating symptoms may obscure the disease, mechanism-based drug development allows the two approaches to converge. Hub proteins, those that coordinate the expression of proteins that mediate specific cellular functions, may be dysregulated across a broad range of disorders. Herein, we show that the RNA-binding protein DJ-1 controls the activity of L-type voltage-dependent calcium channels (L-VDCC), via the expression of its auxiliary subunit alpha2delta2 ( $\alpha 2 \delta 2)$. Importantly, we demonstrate that this novel DJ-1/a2ס2/L-VDCC pathway is commonly disrupted among neurological disorders, namely Alzheimer's disease (AD) and Tuberous Sclerosis (TS). Collectively, these data rationalize mechanism-based drug therapy to treat disease.

\section{Introduction}

The mammalian target of rapamycin (mTOR) signaling pathway regulates the expression of proteins. Neurological disorders that manifest overactive mTOR (mTORopathy) are characterized by neuronal hyperexcitability and seizures, a shared symptom of autism spectrum disorder (ASD) and late-stage Alzheimer's disease (AD) 
(1-4). The best example of a prototypical mTORopathy is tuberous sclerosis complex (TS). Individuals with TS display hyperactive mTOR signaling which is believed to be the root cause of epilepsy and ASD often associated with this disorder $(5,6)$.

Loss of function of either TSC1 or TSC2 protein increases mTOR signaling (7). mTOR forms two complexes-mTORC1 and $\mathrm{C} 2$-and it is well-established that the mTORC1 pathway promotes protein synthesis (8). It was therefore unexpected that a mouse model of TS exhibited reduced protein synthesis (9). Subsequent to this study, AD has also been found to exhibit reduced protein synthesis (10). Since then, compelling questions have arisen but remain unanswered: what mRNAs are repressed in mTORopathies, how are these mRNAs translationally regulated, and what are the functional consequences of repressing these mRNAs? Here, we demonstrate that DJ-1 (also known as Parkinsonism associated deglycase or Parkinson disease (autosomal recessive, early onset) 7 (Park7)), an RNA-binding protein, is a major translational hub disrupted in TS and AD. DJ-1 represses the translation of the calcium $\left(\mathrm{Ca}^{2+}\right)$ channel subunit Cacna $2 d 2 \mathrm{mRNA}$, which encodes $\alpha 2 \delta 2$ protein that facilitates the stability and trafficking of $\mathrm{Ca}^{2+}$ channels. Thus, elevated DJ-1 levels reduce the dendritic expression of $\alpha 2 \delta 2$ and L-type voltage-dependent $\mathrm{Ca}^{2+}$ channel (VDCC) activity. These studies bring to light a novel mechanism of controlling local $\mathrm{Ca}^{2+}$ signaling that is commonly dysregulated in developmental- and aging-associated mTORopathies.

\section{Results}

\section{DJ-1 target identification}


We previously found that DJ-1 is associated with translation regulation and its expression is sensitive to mTORC1 activity in the postsynaptic density (PSD) isolated from the cortex (11). To test the role of DJ-1 as a translational hub that may be disrupted in mTORopathies, we first computationally defined the DJ-1-binding sequence (12). We found that the RNA sequences GNGCNG and CNGCNG are highly represented in known DJ-1-associated mRNAs (Fig. 1A) (12). Because DJ-1 is in the PSD, we sought the number of occurrences of these two DJ-1-binding motifs in mRNAs that encode PSD-associated proteins $(11,13)$. These mRNAs were ranked by frequency of DJ-1-binding sequence per kilobase (freq/kB). Of the 916 PSD-associated mRNAs, the mean freq/kB of both motifs in different mRNA regions are: 1.72 in 3' untranslated region (UTR), 15.66 in cDNA or coding region (CR), and 0.43 in 5'UTR. The distribution of DJ-1-binding motifs throughout the mRNA suggests that DJ-1 may control the elongation step of protein synthesis, consistent with the role that DJ-1 represses its target mRNAs $(12,14,15)$.

DJ-1, prior to our report, has been primarily linked to Parkinson's disease, yet never to epilepsy and ASD—symptoms that are common in TS—or AD (11). Recently, however, it has been identified as a potential biomarker for dementia (16). We reasoned, therefore, that DJ-1's RNA-binding properties and target mRNAs may underlie a common pathology in these disorders. A Gene Ontology (GO) enrichment analysis of putative DJ-1 target mRNAs that encode PSD-associated proteins linked to epilepsy, $A D$, and $A S D$ reveals that many of these targets are involved in ion transport (Table S1 and S2). Consistent with ion transport in the PSD, Grin2a, which codes for an ionotropic glutamate receptor, is the only DJ-1-target ion channel implicated in 
epilepsy, AD, and ASD. (Fig. 1B; Table S2, S3). Since NMDA receptor expression and function have been extensively characterized in these disorders, we decided to focus on DJ-1 targets related to epilepsy since seizures have been suggested to underlie both $A D$ and ASD (17-22). We noted several coding for $\mathrm{Ca}^{2+}$ channels in epilepsy but not in AD or ASD. GO analysis of these mRNAs by disease indeed reveals an enrichment of "calcium ion transport"-associated proteins in epilepsy (Fig. 1C and D; Table S2). We focused on $\mathrm{Ca}^{2+}$-associated channels, because $\mathrm{Ca}^{2+}$ is arguably the most important second messenger in the brain as it is essential in neurodevelopment, synaptic transmission, and plasticity $(23,24)$. Moreover, disrupted $\mathrm{Ca}^{2+}$ homeostasis is suggested to be a crucial turning point leading to the progression of many neurodegenerative diseases (25). We focused on Cacna2d2, which encodes $\alpha 2 \delta 2$, since $\alpha 2 \delta 2$ promotes the forward trafficking of several pore forming $\mathrm{Ca}^{2+}$ channel subunits to increase current density and influence channel activation and inactivation kinetics (26-28). Additionally, loss of function mutations in CACNA2D2 gene can lead to seizure activity, a common symptom in epilepsy, ASD and AD (29). $\alpha 2 \delta$ subunits are typically localized to presynaptic terminals; however, little is known about the subcellular localization of $\alpha 2 \delta 2(30)$. In situ hybridization of Cacna2d2 mRNA surprisingly indicates that it resides in the dendrites_in addition to the soma-intimating that $\alpha 2 \delta 2$ can be synthesized upon changes in synaptic activity (Fig. 2A).

Cacna2d2 mRNA has several predicted DJ-1-binding sites (5'UTR: 15-26; CR: 58-61; 3'UTR: 7-8) (Table S3). To test if Cacna2d2 mRNA is a DJ-1 target, we performed RNA immunoprecipitation (RNA-IP) by isolating both DJ-1 protein and Cacna2d2 mRNA from anti-DJ1 RNA-IP fraction. Western blot analysis demonstrates 
that DJ-1 protein binds to DJ-1 antibody (Fig. 2B). Furthermore, reverse-transcription followed by semi-quantitative polymerase chain reaction (RT-qPCR) detects Cacna2d2 mRNA specifically in DJ-1 and not the negative IgG control (Fig. 2B). These data confirm that Cacna2d2 mRNA is a target of DJ-1. To determine if DJ-1 promotes or represses Cacna2d2 mRNA translation, we knocked down DJ-1 in cultured hippocampal neurons by siRNA and measured $\alpha 2 \delta 2$ protein levels by immunocytochemistry. Knockdown of DJ-1 increases $\alpha 2 \delta 2$ in dendrites by $\sim 60 \%$, confirming a role for DJ-1 as a translational repressor (Fig. 2C) (12).

\section{DJ-1 attenuates L-VDCC activity}

$\alpha 2 \delta$ subunits associate with voltage-dependent $\mathrm{Ca}^{2+}$ channels (VDCC) (26-28). Since perturbing DJ-1 levels alter $\alpha 2 \delta 2$ expression in the dendrites, we predicted that DJ-1 can also affect a dendritic VDCC through $\alpha 2 \delta 2$ levels. Notably, L-type VDCC, which is open at rest, is prominently expressed on dendrites (31). We, therefore, assessed L-type channel function by performing live $\mathrm{Ca}^{2+}$ imaging in dissociated rat hippocampal neurons that overexpress DJ-1. We activated L-type VDCC with its channel opener, BayK-8644 (BayK, $5 \mu \mathrm{M})$ and measured the change in dendritic $\mathrm{Ca}^{2+}$ fluorescence $(\Delta F / F)$. Application of BayK on pcDNA-expressing neurons, which serve as control, increases dendritic fluorescence by 30\% (Fig. 2D). Interestingly, DJ-1overexpressing cells fail to display BayK-induced elevation of $\mathrm{Ca}^{2+}$ signal in dendrites (Fig 2E). These data link DJ-1 to dendritic L-type VDCC function and perhaps the signaling downstream of L-type channels. Through bioinformatics analyses and 
experimental validation, these data collectively establish that $\mathrm{DJ}-1$ can regulate $\mathrm{Ca}^{2+}$ dynamics through $\alpha 2 \delta 2$ expression and L-type VDCC activity.

Aberrant $\alpha 2 \delta 2$ expression and L-VDCC activity in TS

Since dendritic DJ-1 is excessively expressed in a preclinical mouse model of TS, we hypothesized that TS and other mTORopathies have disrupted $\mathrm{Ca}^{2+}$ channel activity (11). We first measured $\alpha 2 \delta 2$ levels, in vivo and in vitro, after knocking out the Tsc1 gene by Cre expression (Tsc1 KO or TS) in dissociated hippocampal neurons and hippocampal slices. Immunostaining for $\alpha 2 \delta 2$ indeed shows that TS neurons have $\sim 30 \%$ lower $\alpha 2 \delta 2$ levels in the dendritic fields of hippocampal CA1 region and in dendrites of cultured hippocampal neurons compared to control (Fig. 3A and B). Low $\alpha 2 \delta 2$ levels predict reduced $\mathrm{Ca}^{2+}$ signaling in TS dendrites (27). To determine if TS dendrites have reduced L-type currents, we activated L-type VDCC with BayK and measured dendritic $\Delta \mathrm{F} / \mathrm{F}$. WT dendrites, as expected, show a $\sim 25 \%$ increase in $\Delta \mathrm{F} / \mathrm{F}$ with BayK (Fig. 3C) $(26,27,30)$. Surprisingly, BayK-induced L-type activity is absent in TS, similar to DJ-1 overexpression (Fig. 2E). These data bring to light that TS harbors abnormal L-type VDCC activity, a newly-identified TS-associated dysfunction.

Aberrant $\alpha 2 \delta 2$ and Cav1.2 expression and L-VDCC activity in $A D$

Do aberrant DJ-1 and $\alpha 2 \delta 2$ levels and L-type channel signaling constitute a molecular signature of a mTORopathy? To answer this question, we used a preclinical 
mouse model of Alzheimer's disease_APP/PS1 transgenic mouse-since aberrant mTOR signaling is observed in AD (2-4). We prepared dissociated hippocampal neurons from APP/PS1 mice and their wildtype littermates. Similar to our preclinical TS mouse model, hippocampal neurons of APP/PS1 mice have $\sim 200 \%$ more dendritic DJ-1 and $\sim 50 \%$ less $\alpha 2 \delta 2$ levels compared to wildtype controls (Fig. 4A and B). Moreover, BayK-induced L-type signaling is attenuated in APP/PS1 dendrites (Fig. 4C and D). In agreement with our prediction, the preclinical mouse model of AD harbors similar

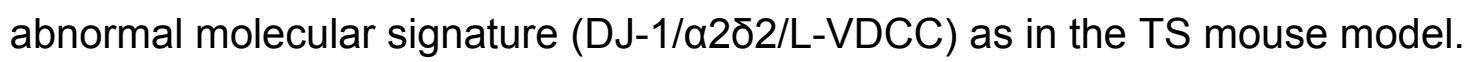

As preclinical models of TS and AD show the same abnormal molecular signature-elevated DJ-1 and diminished $\alpha 2 \delta 2$ levels and L-type VDCC hypoactivitywe sought whether these signs are recapitulated in human AD. Using synaptoneurosomes isolated from human prefrontal cortices (PFC), we first measured mTOR activity in control and AD-diagnosed tissues. Subject gender, age, beta-amyloid load, Braak score, and dementia status are reported (Table S4). Western blot analyses of phospho-S6, a downstream marker of mTOR activity, is significantly elevated by $\sim 200 \%$ in AD samples, consistent with previous reports that mTOR is hyperactive in AD (Fig. 4E) (32-34). We next assessed DJ-1 expression as it is regulated by mTOR activity (11). DJ-1 expression, similar to TS and AD mouse models, is $\sim 50 \%$ more in $A D$ compared to age-matched control samples (Fig. 4F). Because DJ-1 interacts with Cacna2d2 mRNA (Fig. 2B) and abates dendritic $\alpha 2 \delta 2$ levels (Fig. 2C), we measured a2ס2 in human PFC synaptoneurosomes. Recapitulating the observations in AD and TS mouse models, the human AD samples express $\sim 50 \%$ less $\alpha 2 \delta 2$ levels than control (Fig. 4G). L-type VDCC is hypoactive in dendrites of preclinical models of TS and AD. 
As a surrogate for L-type channel activity in postmortem human brain tissue, we hypothesized that the protein expression of Cav1.2-the pore-forming subunit of L-type $\mathrm{Ca}^{2+}$ channels-in AD samples is low compared to control. Cav1.2 expression is indeed $\sim 50 \%$ lower in human brains with $A D$ than controls (Fig. $4 \mathrm{H}$ ). Altogether, these data suggest that TS and AD share dysregulated L-type VDCC-associated protein expression that effect calcium signaling in dendrites.

\section{Discussion}

As clinical trials, to date, have failed or produced mixed results in alleviating cognitive issues in both AD and TS, it is critical to determine the molecular mechanisms that underlie synaptic dysfunction in these disease (35). Here, using an unbiased, bioinformatics approach, to identify new ASD- and AD-related proteins. Notably, an enrichment of epilepsy-related $\mathrm{Ca}^{2+}$ channels that are DJ-1 target mRNAS, justified the exploration of $\mathrm{Ca}^{2+}$ dynamics in other mTORopathies with excessive expression of dendritic DJ-1. This finding also allowed us to uncover a novel L-VDCC pathology shared between TS and AD. Moreover, DJ-1 may impact several $\mathrm{Ca}^{2+}$ associated mRNAs, as predicted by our bioinformatics (Fig. 1) and $\mathrm{Ca}^{2+}$ dysregulation may be a common feature of mTORopathies. These discoveries may lead to the development of new mechanism-based treatments based on synaptic gene expression regulated by a single RBP (see accompanying manuscript).

L-type VDCC has been suggested to be a homeostat for multiple pathways and dysregulated in ASD $(36,37)$. Our data expand the role of L-type VDCC as a central 
homeostat for mTORopathies beyond ASD. The animal models of TS and AD that we use here express elevated DJ-1, low $\alpha 2 \delta 2$, and repressed L-type VDCC activity. L-type VDCCs are critical for normal synaptic function, and altering their activity is linked to neurological disorders, such as seizures, a core symptom of TS and AD $(36,38)$ (see accompanying manuscript). Importantly, L-VDCC blockers are being considered as potential drug therapies (36-38). Our data, however, suggest that blocking L-VDCC may not be suitable for ASD, TS, AD, and perhaps for other mTORopathies, since L-VDCC function in these neurological disorders is already absent or low at best. Moreover, these data collectively may explain why L-VDCC blockade can facilitate subtle, nonconvulsive epilepsy, paralleling what is observed in TS and dementia patients (3942). In summary, our work identifies the DJ-1/ $22 \delta 2 / L-V D C C$ pathway in $A D$ and $T S$, providing new druggable targets for the treatment of these disorders.

\section{Methods}

Determination of DJ-1-binding motifs and disease associated-gene overlap

Using a 45-nucleotide consensus sequence previously identified, we constructed a list of all possible 6-12 nucleotide substrings, a typical length of RNA-binding motifs (12, 4345). Each substring was then ranked based on the sum of its probability values based on the probabilities derived from the consensus logo. We found that two motifs GNGCNG or CNGCNG occurred with highest probability. To find the frequency of occurrences for these motifs in a given DNA alphabet, we used the seqinr and Biostrings packages $(46,47)$. The graphical user interface for this process was 
developed using the shiny package in $\mathrm{R}$ along with the DT package $(48,49)$. PSD gene data were obtained from the BAYES-COLLINS-MOUSE-PSD-CONSENSUS available online at G2C: Genes to Cognition database (http://www.genes2cognition.org/) (13). All gene sequences were obtained using the biomart package (50-52). To create the Venn diagram, we first determined the frequency per kilobase (freq/kB) of putative DJ-1 RNAbinding motifs (GNGCNG or CNGCNG) within the 5' UTR, cDNA, and 3' UTR sequences of the 916 PSD genes with available sequence data. All matches were filtered to obtain only the maximum length sequences and then ranked by freq/kB in descending order. This list was then subset based on the overlap with mTOR-regulated proteins associated with epilepsy, Alzheimer's disease, and autism spectrum disorder. Finally, the lists were thresholded using Grin2a as a cutoff and generated the Venn diagram. Disease-associated mTOR-regulated genes were obtained from databases as described by Niere et al, 2016 (11).

\section{$\underline{\text { Mice }}$}

\section{Tsc1 conditional knockout}

For the TSC mouse model, Tsc $1^{t m 1 D j k} / J$ mice (Jackson Laboratory, Bar Harbor, ME) were used (53). To generate Tsc1 KO, recombinant adeno-associated virus coding for Cre recombinase (AAV-Cre-GFP) was introduced in $T s c 1^{t m 1 D j k} / \mathrm{J}$ mice to knockout the tsc1 gene, and AAV-GFP (vector) served as control(11). Both AAV-GFP and AAVCRE-GFP were driven by a synapsin promoter. As previously described for in vivo studies, AAV-Cre-GFP or AAV-GFP was injected into the hippocampus of 7 to 8 weeksold male mice using the following coordinates (from bregma): $\pm 2.2 \mathrm{~mm} \mathrm{~A} / \mathrm{P}, \pm 1.5 \mathrm{~mm}$ 
$\mathrm{M} / \mathrm{L} ; \pm 2.5 \mathrm{~mm} \mathrm{~A} / \mathrm{P}$, and $\pm 1.6 \mathrm{~mm} \mathrm{M} / \mathrm{L}(11)$. Two weeks after introducing one of the two viruses, hippocampal slices were generated and used for experiments. Male and female mice were used to generate dissociated hippocampal neurons.

\section{APP/PS1}

For the AD animal model, APP/PS1 mouse line which expresses human/murine amyloid precursor protein (APP) construct containing the Swedish double mutation (APPswe)

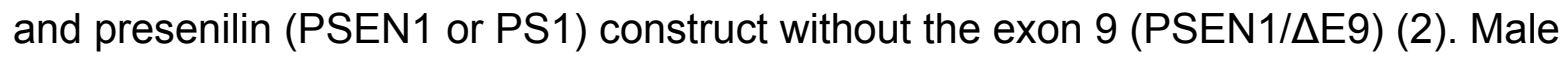
and female mice were used, and WT littermates served as control for studies involving APP/PS1.

\section{$\underline{\text { Cell culture }}$}

Dissociated hippocampal neurons, 18-25 days in vitro (DIV), were prepared from embryonic rat pups (E17-18) similar to Sosanya et al (54). For Tsc1 or APP/PS1 cultures, neurons were prepared from postnatal (0-3 days) Tsc1 conditional knockout or APP/PS1 pups similar to Niere et al (55).Briefly, hippocampi were extracted from postnatal day 1-3 Tsc $1^{t m 1 D j k / J}$ or APP/PS1 mouse pups. The tissue was dissociated and plated in neurobasal A medium supplemented with B27, glutamine, and $1 \%$ fetal bovine serum. Cultures were plated at a density of $\sim 100,000$ cells per $12 \mathrm{~mm}$ on glass coverslips that had been coated overnight with $50 \mu \mathrm{g} \mathrm{ml}^{-1}$ poly-D-lysine and $25 \mathrm{\mu g} \mathrm{ml}^{-1}$ laminin in borate buffer. Cultures were fed after 1 day after plating, and media was replaced approximately once a week with either fresh rat culture media (neurobasal A supplemented with B27, glutamine and $3 \mu \mathrm{M}$ cytosine arabinoside 
$(\mathrm{AraC})$ ) or fresh mouse culture media (glial-conditioned media with $3 \mu \mathrm{M} \mathrm{AraC}$ ) until cultures were used at DIV 14-21.

\section{Fluorescence in situ hybridization (FISH)}

Cacna2d2 mRNA detection was conducted using the ViewRNA ISH Cell Assay kit (Affymetrix) as described in Cajigas et al, 2012 (56). The Cacna2d2 probe set was designed commercially by Affymetrix. Briefly, primary hippocampal neurons (days in vitro (DIV) 20-21) were fixed at room temperature for 30 minutes with a $4 \%$ paraformaldehyde solution (4\% paraformaldehyde, $5.4 \%$ glucose, $0.01 \mathrm{M}$ sodium metaperiodate, in lysine-phosphate buffer). Proteinase $\mathrm{K}$ treatment was omitted and the rest of the hybridization was completed according to the manufacturer's instructions. The cells were then washed with PBS and blocked with $4 \%$ goat serum in PBS for one hour followed by incubation in primary antibody (chicken anti-GFP) overnight at $4^{\circ} \mathrm{C}$. After three washes with PBS the cells were incubated with the appropriate secondary antibody for one hour at room temperature and washed with PBS. The coverslips were then mounted with an antifading mounting medium and imaged as described above.

\section{RNA immunoprecipitation (RIP)}

Mouse hippocampal tissue (pooled hippocampus from 6 mice) was homogenized in PLB buffer and incubated for 10 minutes on ice. The samples were then centrifuged for $14,000 \mathrm{xg}$ for 15 minutes at $4^{\circ} \mathrm{C}$. Protein concentration of the supernatant was determined with a BCA assay. The supernatant was then precleared with $5 \mu \mathrm{g}$ goat 
$\operatorname{lgG}$ and $50 \mu \mathrm{l}$ beads (Protein $\mathrm{G}$ ) for 30 minutes at $4^{\circ} \mathrm{C}$. Beads washed with NT2 buffer were incubated with either $30 \mu \mathrm{g}$ DJ-1 antibody (Santa Cruz) or goat IgG for 23 hours at $4^{\circ} \mathrm{C}$. The beads were washed 3 times with NT2 buffer. The precleared samples $(4.5 \mathrm{mg})$ were incubated with the conjugated beads overnight at $4^{\circ} \mathrm{C}$. The beads were washed 4 times with NT2 buffer and after the last wash were suspended in $200 \mu \mathrm{l} \mathrm{NT2} \mathrm{buffer.} 50 \mu \mathrm{l}$ of the bead suspension was removed and incubated with 2X Laemmli sample buffer containing BME for 30 minutes at room temperature. The supernatant was then used for western blot analysis to confirm DJ-1 protein was immunoprecipitated. The rest of the bead suspension in NT2 buffer was used for RNA extraction. The supernatant was removed and RNA extraction was performed with TRIzol. A portion of the eluted RNA was used for quality assessment using an Agilent Bioanalyzer. Reverse transcription was performed with the iScript cDNA synthesis kit (Bio-Rad) according to the manufacturer's instructions. PCR amplification was performed with commercially available gene specific primers for Akt and Cacna2d2 (GeneCopoeia) and PCR products were then run on a 2\% agarose gel.

\section{$\underline{\text { Transfection }}$}

Primary hippocampal neurons were transiently transfected on DIV 14 with siRNA directed against DJ-1(SMARTpool: ON-TARGETplus DJ-1 siRNA, Cat No. L-05018101-0005, Dharmacon) or scrambled siRNA (Dharmacon) plus a tdTomato plasmid; DJ-1 (pGEX-5X-1-DJ1-WT; Addgene) or pcDNA plus RFP plasmid(57) Hippocampal cultures were transfected with Lipofectamine 2000 (Invitrogen) according to the 
manufacturer's instructions. Transfection was allowed to occur for 2.5 hours. Cells were processed for immunohistochemistry on DIV18.

$\underline{\text { Immunohistochemistry (IHC) }}$

For hippocampal slices, mice were transcardially perfused with phosphate buffered saline $(0.1 \mathrm{M})$. Brains were removed and postfixed in $4 \%$ paraformaldehyde. $50 \mu \mathrm{m}$ thick slices were prepared and checked for GFP expression. Slices expressing GFP were processed for IHC, similar to Niere et al, using the following primary antibodies: rabbit anti- $\alpha 2 \delta 2$ (1:100, Novus), mouse anti-MAP2 (1:500, Abcam), and chicken antiGFP (1:500, Aves) (55). With dissociated hippocampal neurons, cells were fixed in 4\% paraformaldehyde for 20 minutes at room temperature and permeabilized in $0.2 \%$ Triton $\mathrm{X}-100$ for 10 minutes (55). Fixed cells were incubated overnight in $4^{\circ} \mathrm{C}$ with the following primary antibodies: rabbit anti- $\alpha 2 \delta 2$ (1:500, Alomone) or rabbit anti-DJ-1 (1:1000, Novus), and chicken anti-Map2 (1:1000, Aves) or mouse anti-Map2 (1:1000, Abcam). Appropriate secondary antibodies (1:500, Life Technologies) were usedAlexaFluor488 (AF488), AF647, AF405-after overnight primary antibody incubation.

\section{$\underline{\text { Calcium imaging }}$}

Dissociated hippocampal neurons at DIV 14-21 were used for live calcium imaging. Prior to imaging, cells were incubated in ACSF with Oregon Green 488 BAPTA-1 AM (OGB, $200 \mu \mathrm{M}$; 30 minutes; $37^{\circ} \mathrm{C}$; ThermoFisher) as described by Workman et al (58). Cells were then transferred to fresh ACSF $\left(37^{\circ} \mathrm{C}\right)$ for imaging $(1$ frame per 20 seconds). Baseline calcium signal was imaged (1 minute), after which (S)-(-)-Bay- 
K-8644 ( $5 \mu \mathrm{M}$, Tocris) or vehicle (DMSO) was added. Neurons were imaged for $\sim 600 \mathrm{~s}$ at room temperature. Quantification of the calcium signal was performed using Metamorph (Molecular Devices). Briefly, dendritic regions of interest (ROI) that were at least $5 \mu \mathrm{m}$ from the soma were analyzed. The mean intensity values for each ROI at every 20 seconds were averaged as baseline $\left(F_{0}\right)$. The ROI intensity values obtained at each time point after the addition of BayK-8644 or vehicle were averaged $(F)$. The equation, $\Delta F / F=\left(\left(F-F_{0}\right) / F_{0}\right)$, was used to measure the change in signal and data were plotted as a percentage of the baseline (58).

\section{$\underline{\text { Western blots }}$}

\section{Subjects and Tissues}

Postmortem human prefrontal cortex (PFC) tissue samples were acquired from the University of Washington (UW) Neuropathology Core in accordance with the UW and Wake Forest University School of Medicine Institutional Review Boards. Tissues were harvested from patients that were clinically diagnosed with $A D$, where the neuropathology of $A D$ was confirmed and from controls who exhibited low levels of $A D$ neuropathology. The samples in this study underwent rapid autopsy shortly after death, where the harvested tissue was flash frozen. Clinical and neuropathological diagnoses were based on postmortem senile plaques, Braak neurofibrillary tangle staging and Consortium to Establish a Registry for Alzheimer's Disease (CERAD) scores, collectively known as the "ABC score". The patient characteristics are shown in Table S4. Mean age of death is 86.9 years, and the postmortem interval (PMI) ranged between 2 and 5 hours with an average of 4.03 hours. 


\section{Synaptoneurosome preparation}

Frozen postmortem human prefrontal cortex samples from UW Neuropathology Core were homogenized with lysis buffer composed of Tris-Base with Halt ${ }^{\mathrm{TM}}$ Phosphatase and Protease Inhibitor Cocktail (Thermo Scientific). Homogenates were then sequentially filtered through $100 \mu \mathrm{m}$ cell strainer and $5 \mu \mathrm{m}$ syringe filter and centrifuged for 20 minutes in $4^{\circ} \mathrm{C}$ at $14,000 \mathrm{xg}$. The pellet was solubilized with radioimmunoprecipitation (RIPA) buffer and centrifuged for 10 minutes in $4^{\circ} \mathrm{C}$ at 14,000 $\mathrm{xg}$. The supernatant was collected for protein quantification using Pierce ${ }^{\mathrm{TM}} \mathrm{BCA}$ Protein Assay Kit (Thermo Scientific).

Western blot analysis

Samples were prepared into SDS-polyacrylamide gel electrophoresis (PAGE) loading buffer using 4x Laemmli Sample Buffer (Bio-Rad). $50 \mu g$ protein from each sample were resolved in $10 \%$ SDS-polyacrylamide gel and transferred onto $0.2 \mu \mathrm{m}$ nitrocellulose membranes (Bio-Rad). The nitrocellulose membranes were blocked in $5 \%$ nonfat dry milk in Tris-buffered saline containing $0.1 \%$ Tween 20 . To visualize the proteins, we used: mouse anti-DJ1 (1:2000; Novus Biologicals), mouse anti-tubulin (1:20,000; Abcam), rabbit anti- $\alpha 2 \delta 2$ (1:5000; Alomone Labs, Jerusalem, Israel), mouse antiribosomal S6 (1:1000, Cell Signaling), rabbit anti-phospho-S6 (1:1000, Cell Signaling), and mouse anti-Cav1.2 (1:2000; Neuromab). Antibody-bound membranes were incubated in fluorescence-conjugated secondary antibodies (AF680, Life Technologies; AF800, LiCor; 1:5000). Fluorescent images of the membranes were obtained using the Odyssey CLx infrared imaging system. Densitometry analyses of proteins were conducted using ImageJ (National Institutes of Health) software. 


\section{Acknowledgments}

This study was supported by National Institutes of Health NINDS NS105005 (KRG); the National Science Foundation IOS 1026527 and IOS 1355158 (KRG), Postdoctoral Research Fellowship in Biology DBI-1306528 (FN) and DBI-1103738 (LPC); Alzheimer's Association AARF-19-614794 (FN); Department of Defense, United States Army Medical Research and Materiel Command USAMRMC Award W81XWH-14-10061 and W81XWH-19-1-0202 (KRG). National Institute of Aging Wake Forest School of Medicine Alzheimer's Disease Research Center Pilot Grant P30AG049638 (KRG)

Author contributions: FN, LPC, AU, SC, CDK, TM and KRG designed research. FN, LPC, AU, SN, CR, JP and WCT conducted experiments and analyzed data. CDK and SC provided the human samples. FN, LPC, AU, SN, and KRG wrote the manuscript.

\section{Figure Legend}

Figure 1. Autism spectrum disorder (ASD)-, epilepsy-, and Alzheimer's disease (AD)risk mRNAs contain putative DJ-1-binding motifs. (A) Putative DJ-1-binding sequence. (B) Venn diagram depicting DJ-1 target mRNAs whose proteins associate with the postsynaptic density (PSD). The mRNAs are grouped based on (1) the location of DJ-1binding motifs in the sequence, namely 5' untranslated region (UTR), coding region (cDNA) or 3'UTR, and (2) their linkage to ASD, epilepsy, and/or AD. (C) Percentages of risk mRNAs for ASD, $A D$, and epilepsy that encode $\mathrm{Ca}^{2+}$ channel-associated proteins and contain DJ-1-binding motifs . 10, 13 and 27 percent of DJ-1-target mRNAs linked to 
ASD, AD and epilepsy, respectively, participate in " $\mathrm{Ca}^{2+}$ ion transport" (Table S2). (D) List of DJ-1-target mRNAs whose proteins are implicated in " $\mathrm{Ca}^{2+}$ ion transport".

Figure 2. DJ-1 regulates the dendritic expression of $\alpha 2 \delta 2$ and activity of L-type VDCC. (A-C) Characterization of Cacna2d2 mRNA, which encodes $\alpha 2 \delta 2$, as a DJ-1 target. A. (top) Representative fluorescence in situ hybridization of Cacna2d2 mRNA depicting its presence in soma and dendrites. (Bottom) Magnified images of dendrites (outlined by broken lines) from top panel containing Cacna2d2 mRNA (green dots/white arrowheads). (B) Co-immunoprecipitation of DJ-1 and Cacna2d2 mRNA. (Top) Representative immunoblot of DJ-1 coimmunoprecipitating with anti-DJ-1 antibody. (Bottom) RT-qPCR-amplified product of Cacna2d2 mRNA isolated from DJ-1 immunoprecipitation. (C) Knockdown of DJ-1 increases $\alpha 2 \delta 2$ protein. (Left) Representative images of $\alpha 2 \delta 2$ protein (fire/white arrows) in neurons (top) expressing scrambled (control/Ctl) and (bottom) DJ-1/knockdown siRNAs. Map2 marks dendrites. (Right) Quantification of dendritic $\alpha 2 \delta 2$ normalized to Ctl. Ctl: $1.00 \pm 0.11, \mathrm{n}=23$; DJ-1: $1.58 \pm 0.32 \mathrm{n}=6$. (D and E) Increased expression of DJ-1 inhibits L-type VDCC activity. Assessment of dendritic L-type $\mathrm{Ca}^{2+}$ channel activity in (D) pcDNA-expressing and (E) DJ-1-overexpressing dissociated hippocampal neurons using L-type agonist, BayK-644 (BayK, $5 \mu \mathrm{M}$ ). (Top) Average trace of $\mathrm{Ca}^{2+}$ fluorescence signal before (baseline; 0-60 seconds (sec)) and after (520-580 sec) addition of BayK or vehicle at $\sim 90 \mathrm{sec}$. (Bottom) Quantification of change in fluorescence $(\Delta F)$ normalized to baseline $(F)$. (D) BayK, but not vehicle (Veh), increases $\mathrm{Ca}^{2+}$ fluorescence in pcDNA-expressing dendrites. Veh:

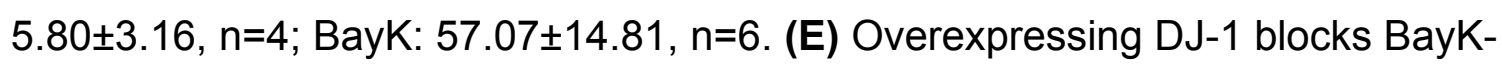


induced increase in $\mathrm{Ca}^{2+}$ fluorescence. Veh: $-3.10 \pm 6.04, \mathrm{n}=17$; BayK: 13.44 $\pm 7.57, \mathrm{n}=11$.

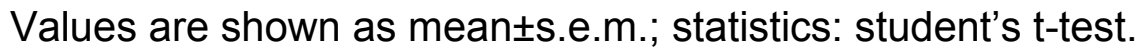

Figure 3. Preclinical models of TS exhibit diminished $\alpha 2 \delta 2$ expression and L-type VDCC activity. (A) (Top) Compared to wildtype mice expressing GFP alone (WT), (bottom) Cre-GFP-mediated knockdown of Tsc1 (TS) in the hippocampus reduces basal $\alpha 2 \delta 2$ levels in stratum radiatum of CA1 area as shown by immunohistochemistry of acute slices. GFP indicates Cre expression and Map2 marks dendrites. Quantification normalized to WT (WT: 1.00 $\pm 0.01, \mathrm{n}=12$; TS: $0.72 \pm 0.07 \mathrm{n=12}$ ). (B) (Top) Basal expression of $\alpha 2 \delta 2$ in dendrites of WT dissociated hippocampal neurons is significantly higher than (bottom) that of Cre-GFP-expressing neurons (TS) as shown by immunocytochemistry. Map2 marks dendrites. Quantification normalized to WT (WT: 1.00 $\pm 0.04, n=37 ;$ TS: $0.70 \pm 0.08 n=19)$. (C and D) L-type VDCC activity is absent in TS. (C) While activation of L-type channels by BayK increases $\mathrm{Ca}^{2+}$ fluorescence in WT dendrites (Veh: $-0.96 \pm 1.12, n=20$; BayK: 27.92 $\pm 6.39, n=14),(D)$ BayK fails to increase $\mathrm{Ca}^{2+}$ signal in TS (Veh: $-3.82 \pm 2.98, \mathrm{n}=28$; BayK: $\left.-2.29 \pm 2.44, \mathrm{n}=18\right)$. Values are shown as mean \pm s.e.m.; statistics: student's t-test

Figure 4. The APP/PS1 preclinical model of and human brains with AD exhibit disrupted DJ-1 and L-type-VDCC-associated protein expression and diminished L-type VDCC activity. (A and B) Immunocytochemical analyses in dissociated hippocampal neurons from littermates expressing normal (WT; top image) and mutant APP and PS1 genes (APP/PS1; bottom image) demonstrate that (A) DJ-1 expression (blue) in WT 
dendrites is markedly lower than APP/PS1 as quantified and normalized to WT (WT:

1.00 $\pm 0.23, n=27 ;$ APP/PS1: 2.24 $\pm 0.26, n=30 ; p<0.001$ ); and (B) $\alpha 2 \delta 2$ level (blue) in WT dendrites is higher than APP/PS1, as quantified and normalized to WT (WT: 1.00 \pm 0.17 , n=30; APP/PS1: 0.48 $\pm 0.04, n=30)$. (C and D) L-type $\mathrm{Ca}^{2+}$ channel activity is attenuated in APP/PS1 model of AD. (C) In dissociated WT hippocampal neurons, BayK but not vehicle increases dendritic $\mathrm{Ca}^{2+}$ fluorescence. Veh: 1.22 $\pm 2.81, \mathrm{n}=6$; BayK: 57.29 \pm 6.65 , $\mathrm{n}=5$. (D) Neurons from APP/PS1 littermates display reduced $\mathrm{Ca}^{2+}$ fluorescence in

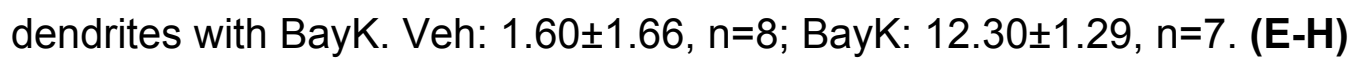
Synaptoneurosomes of human brains diagnosed with AD present mTOR- and L-VDCCassociated protein changes similar to mouse models of TS and AD. (Top) Representative western blots and (bottom) quantification are shown. (E-F) Compared and normalized to age-matched controls (Ctl), AD samples have overactive mTOR as measured by elevated (E) phospho-S6 (Ctl: 1.00 $\pm 0.07, n=3 ; A D: 1.94 \pm 0.34, n=3)$ and

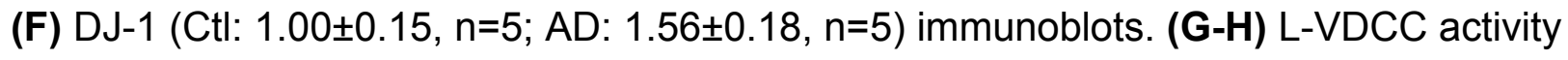
may be attenuated in $A D$ since the following levels of L-type-associated proteins are

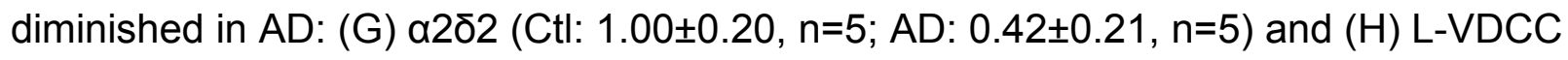
pore-forming subunit Cav1.2 (1.00 $\pm 0.15, n=4 ; A D: 0.45 \pm 0.20, n=3)$. Values are shown

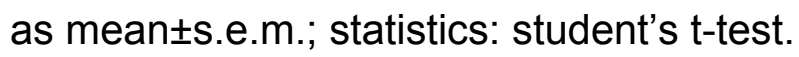




\section{Reference}

1. Crino PB (2015) mTOR signaling in epilepsy: insights from malformations of cortical development. Cold Spring Harbor perspectives in medicine 5(4).

2. Jankowsky JL, et al. (2004) Mutant presenilins specifically elevate the levels of the 42 residue beta-amyloid peptide in vivo: evidence for augmentation of a 42specific gamma secretase. Hum Mol Genet 13(2):159-170.

3. Cai Z, Chen G, He W, Xiao M, \& Yan LJ (2015) Activation of mTOR: a culprit of Alzheimer's disease? Neuropsychiatric disease and treatment 11:1015-1030.

4. Talboom JS, Velazquez R, \& Oddo S (2015) The mammalian target of rapamycin at the crossroad between cognitive aging and Alzheimer's disease. NPJ aging and mechanisms of disease 1:15008.

5. Northrup H, Krueger DA, \& International Tuberous Sclerosis Complex Consensus G (2013) Tuberous sclerosis complex diagnostic criteria update: recommendations of the 2012 linternational Tuberous Sclerosis Complex Consensus Conference. Pediatric neurology 49(4):243-254.

6. Curatolo P, Moavero R, \& de Vries PJ (2015) Neurological and neuropsychiatric aspects of tuberous sclerosis complex. Lancet Neurol 14(7):733-745.

7. Davis PE, Peters JM, Krueger DA, \& Sahin M (2015) Tuberous Sclerosis: A New Frontier in Targeted Treatment of Autism. Neurotherapeutics 12(3):572-583.

8. Saxton RA \& Sabatini DM (2017) mTOR Signaling in Growth, Metabolism, and Disease. Cell 168(6):960-976.

9. Auerbach BD, Osterweil EK, \& Bear MF (2011) Mutations causing syndromic autism define an axis of synaptic pathophysiology. Nature 480(7375):63-68.

10. Ma T, et al. (2013) Suppression of elF2alpha kinases alleviates Alzheimer's disease-related plasticity and memory deficits. Nat Neurosci 16(9):1299-1305.

11. Niere F, et al. (2016) Analysis of Proteins That Rapidly Change Upon Mechanistic/Mammalian Target of Rapamycin Complex 1 (mTORC1) Repression Identifies Parkinson Protein 7 (PARK7) as a Novel Protein Aberrantly Expressed in Tuberous Sclerosis Complex (TSC). Mol Cell Proteomics 15(2):426-444.

12. van der Brug MP, et al. (2008) RNA binding activity of the recessive parkinsonism protein DJ-1 supports involvement in multiple cellular pathways. Proc Natl Acad Sci U S A 105(29):10244-10249.

13. Bayes A, et al. (2012) Comparative study of human and mouse postsynaptic proteomes finds high compositional conservation and abundance differences for key synaptic proteins. PLoS One 7(10):e46683.

14. Darnell JC, et al. (2011) FMRP stalls ribosomal translocation on mRNAs linked to synaptic function and autism. Cell 146(2):247-261.

15. Ascano M, Jr., et al. (2012) FMRP targets distinct mRNA sequence elements to regulate protein expression. Nature 492(7429):382-386.

16. Ohrfelt A, et al. (2016) Increased Cerebrospinal Fluid Levels of Ubiquitin Carboxyl-Terminal Hydrolase L1 in Patients with Alzheimer's Disease. Dementia and geriatric cognitive disorders extra 6(2):283-294.

17. Vossel KA, et al. (2013) Seizures and epileptiform activity in the early stages of Alzheimer disease. JAMA neurology 70(9):1158-1166. 
18. Horvath A, Szucs A, Barcs G, Noebels JL, \& Kamondi A (2016) Epileptic Seizures in Alzheimer Disease: A Review. Alzheimer disease and associated disorders 30(2):186-192.

19. Vossel KA, et al. (2016) Incidence and impact of subclinical epileptiform activity in Alzheimer's disease. Ann Neurol 80(6):858-870.

20. Capal JK, et al. (2017) Influence of seizures on early development in tuberous sclerosis complex. Epilepsy \& behavior : E\&B 70(Pt A):245-252.

21. Srivastava S \& Sahin M (2017) Autism spectrum disorder and epileptic encephalopathy: common causes, many questions. Journal of neurodevelopmental disorders 9:23.

22. Jeste SS \& Tuchman R (2015) Autism Spectrum Disorder and Epilepsy: Two Sides of the Same Coin? Journal of child neurology 30(14):1963-1971.

23. Clapham DE (2007) Calcium signaling. Cell 131(6):1047-1058.

24. Berridge MJ (1998) Neuronal calcium signaling. Neuron 21(1):13-26.

25. Marambaud P, Dreses-Werringloer U, \& Vingtdeux V (2009) Calcium signaling in neurodegeneration. Mol Neurodegener 4:20.

26. Dolphin AC (2012) Calcium channel auxiliary alpha2delta and beta subunits: trafficking and one step beyond. Nat Rev Neurosci 13(8):542-555.

27. Dolphin AC (2016) Voltage-gated calcium channels and their auxiliary subunits: physiology and pathophysiology and pharmacology. J Physiol 594(19):53695390.

28. Gao B, et al. (2000) Functional properties of a new voltage-dependent calcium channel alpha(2)delta auxiliary subunit gene (CACNA2D2). J Biol Chem 275(16):12237-12242.

29. Barclay J, et al. (2001) Ducky mouse phenotype of epilepsy and ataxia is associated with mutations in the Cacna2d2 gene and decreased calcium channel current in cerebellar Purkinje cells. J Neurosci 21(16):6095-6104.

30. Dolphin AC (2013) The alpha2delta subunits of voltage-gated calcium channels. Biochim Biophys Acta 1828(7):1541-1549.

31. Magee JC, Avery RB, Christie BR, \& Johnston D (1996) Dihydropyridinesensitive, voltage-gated $\mathrm{Ca} 2+$ channels contribute to the resting intracellular Ca2+ concentration of hippocampal CA1 pyramidal neurons. J Neurophysiol 76(5):3460-3470.

32. An WL, et al. (2003) Up-regulation of phosphorylated/activated p70 S6 kinase and its relationship to neurofibrillary pathology in Alzheimer's disease. Am J Pathol 163(2):591-607.

33. Li X, Alafuzoff I, Soininen H, Winblad B, \& Pei JJ (2005) Levels of mTOR and its downstream targets 4E-BP1, eEF2, and eEF2 kinase in relationships with tau in Alzheimer's disease brain. FEBS J 272(16):4211-4220.

34. Oddo $S$ (2012) The role of $\mathrm{mTOR}$ signaling in Alzheimer disease. Frontiers in bioscience 4:941-952.

35. Mehta D, Jackson R, Paul G, Shi J, \& Sabbagh M (2017) Why do trials for Alzheimer's disease drugs keep failing? A discontinued drug perspective for 2010-2015. Expert opinion on investigational drugs 26(6):735-739. 
36. Mullins C, Fishell G, \& Tsien RW (2016) Unifying Views of Autism Spectrum Disorders: A Consideration of Autoregulatory Feedback Loops. Neuron 89(6):1131-1156.

37. Zamponi GW (2016) Targeting voltage-gated calcium channels in neurological and psychiatric diseases. Nature reviews. Drug discovery 15(1):19-34.

38. Nanou E \& Catterall WA (2018) Calcium Channels, Synaptic Plasticity, and Neuropsychiatric Disease. Neuron 98(3):466-481.

39. Lin JW, et al. (2000) Distinct molecular mechanisms and divergent endocytotic pathways of AMPA receptor internalization. Nat Neurosci 3(12):1282-1290.

40. van Luijtelaar EL, Ates N, \& Coenen AM (1995) Role of L-type calcium channel modulation in nonconvulsive epilepsy in rats. Epilepsia 36(1):86-92.

41. Noebels J (2011) A perfect storm: Converging paths of epilepsy and Alzheimer's dementia intersect in the hippocampal formation. Epilepsia 52 Suppl 1:39-46.

42. Shehata HS, et al. (2017) Clinical patterns and outcomes of status epilepticus in patients with tuberous sclerosis complex. Therapeutics and clinical risk management 13:779-785.

43. Benoit Bouvrette LP, Bovaird S, Blanchette M, \& Lecuyer E (2020) oRNAment: a database of putative RNA binding protein target sites in the transcriptomes of model species. Nucleic Acids Res 48(D1):D166-D173.

44. Dominguez D, et al. (2018) Sequence, Structure, and Context Preferences of Human RNA Binding Proteins. Mol Cell 70(5):854-867 e859.

45. Li X, Kazan H, Lipshitz HD, \& Morris QD (2014) Finding the target sites of RNAbinding proteins. Wiley interdisciplinary reviews. RNA 5(1):111-130.

46. Charif D \& Lobry JR (2007) SeqinR 1.0-2: A Contributed Package to the R Project for Statistical Computing Devoted to Biological Sequences Retrieval and Analysis. Structural Approaches to Sequence Evolution, eds Bastolla U, Porto M, Roman HE, \& M V (Springer, Berlin ; New York), pp xix, 367 p.

47. Pages H, Aboyoun P, Gentleman R, \& Debroy S (2016) Biostrings: String objects representing biological sequences, and matching algorithms. $R$ package version 2.42.1.

48. Chang W, Cheng J, Allaire JJ, Xie Y, \& Mcpherson J (2016) shiny: Web Application Framework for R. $R$ package version 0.14.2.

49. Xie Y (2016) DT: A Wrapper of the JavaScript Library 'DataTables'. $R$ package version 0.2 .

50. Durinck S, et al. (2005) BioMart and Bioconductor: a powerful link between biological databases and microarray data analysis. Bioinformatics 21(16):34393440.

51. Durinck S, Spellman PT, Birney E, \& Huber W (2009) Mapping identifiers for the integration of genomic datasets with the R/Bioconductor package biomaRt. Nat Protoc 4(8):1184-1191.

52. Smedley D, et al. (2015) The BioMart community portal: an innovative alternative to large, centralized data repositories. Nucleic Acids Res 43(W1):W589-598.

53. Kwiatkowski DJ, et al. (2002) A mouse model of TSC1 reveals sex-dependent lethality from liver hemangiomas, and up-regulation of p70S6 kinase activity in Tsc1 null cells. Hum Mol Genet 11(5):525-534. 
54. Sosanya NM, et al. (2013) Degradation of high affinity HuD targets releases Kv1.1 mRNA from miR-129 repression by mTORC1. J Cell Biol 202(1):53-69.

55. Niere F, Wilkerson JR, \& Huber KM (2012) Evidence for a fragile X mental retardation protein-mediated translational switch in metabotropic glutamate receptor-triggered Arc translation and long-term depression. J Neurosci 32(17):5924-5936.

56. Cajigas IJ, et al. (2012) The local transcriptome in the synaptic neuropil revealed by deep sequencing and high-resolution imaging. Neuron 74(3):453-466.

57. Blackinton J, et al. (2005) Effects of DJ-1 mutations and polymorphisms on protein stability and subcellular localization. Brain Res Mol Brain Res 134(1):7683.

58. Workman ER, Niere F, \& Raab-Graham KF (2013) mTORC1-dependent protein synthesis underlying rapid antidepressant effect requires GABABR signaling. Neuropharmacology 73:192-203. 


\section{GNGCNG \\ CNGCNG}

B

\section{Predicted DJ-1 targets}
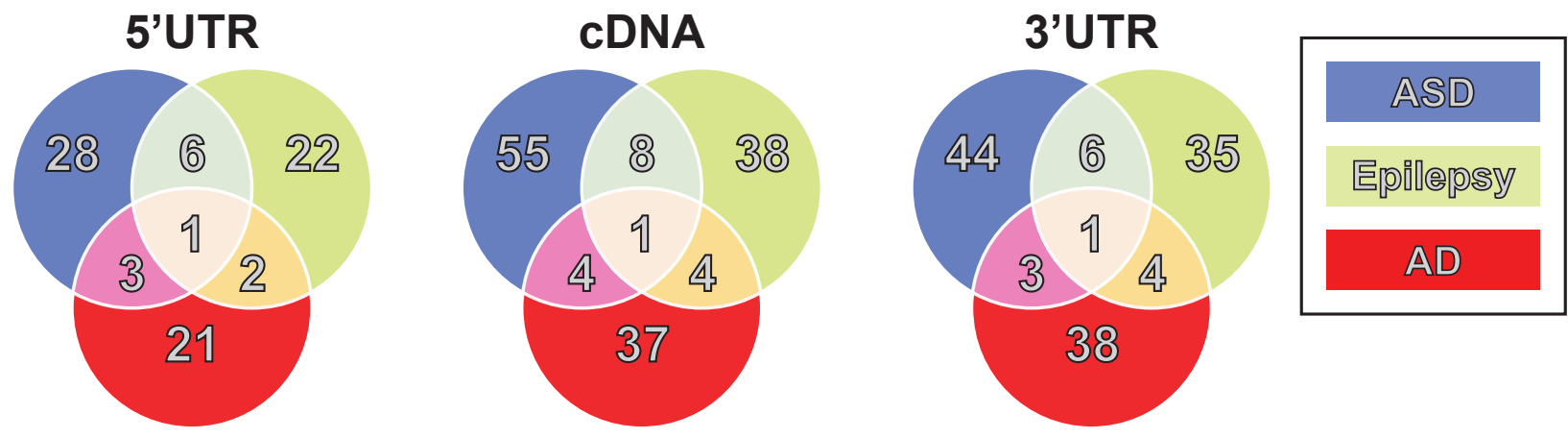

C

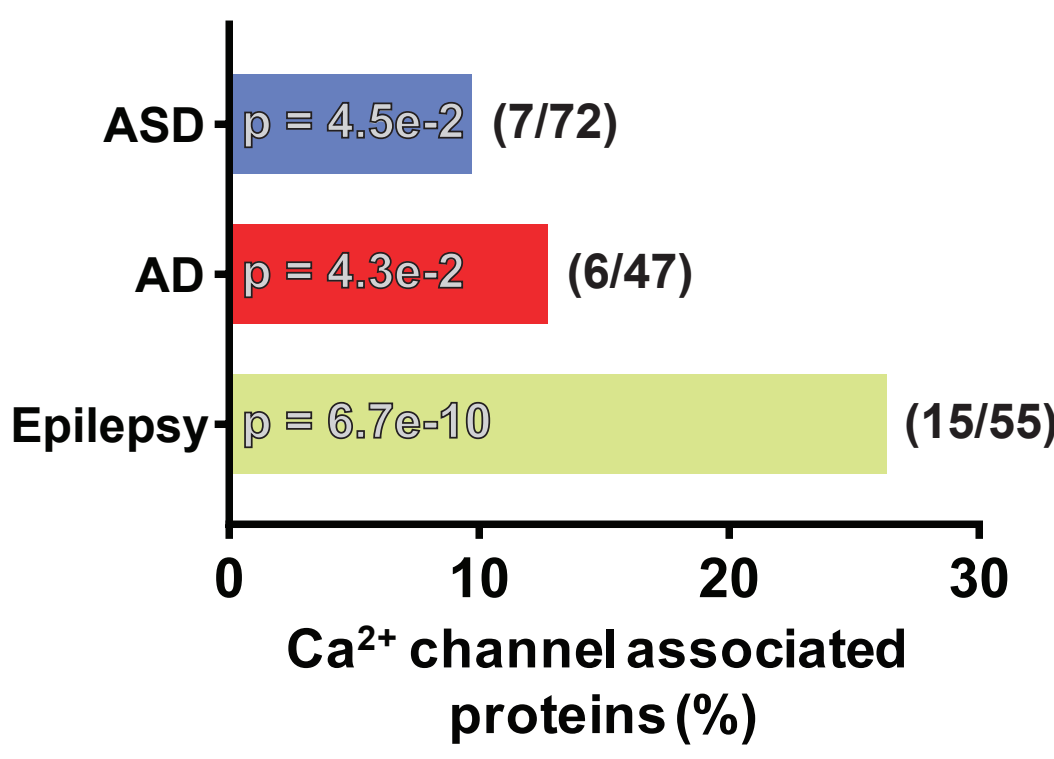

D

\begin{tabular}{|c|c|c|c|c|c|}
\hline \multicolumn{2}{|c|}{ ASD } & \multirow{2}{*}{$\frac{A D}{\text { Atp2a2 }}$} & \multicolumn{3}{|c|}{ Epollepsy } \\
\hline Atp2b2 & Homer1 & & Atp1a2 & Cacna1e & Grin2a \\
\hline Ank2 & & Cdk5 & Fyn & Camk2a & Homer1 \\
\hline Cacna1b & & Grin1 & Cacna2d2 & Camk2d & Ryr2 \\
\hline Ctnnb1 & & Grin2a & Cacnb4 & Cdk5 & \\
\hline Fmr1 & & Nos1 & Cacng3 & Fmr1 & \\
\hline Grin2a & & Рpp3ca & Cacna1a & Grin1 & \\
\hline
\end{tabular}



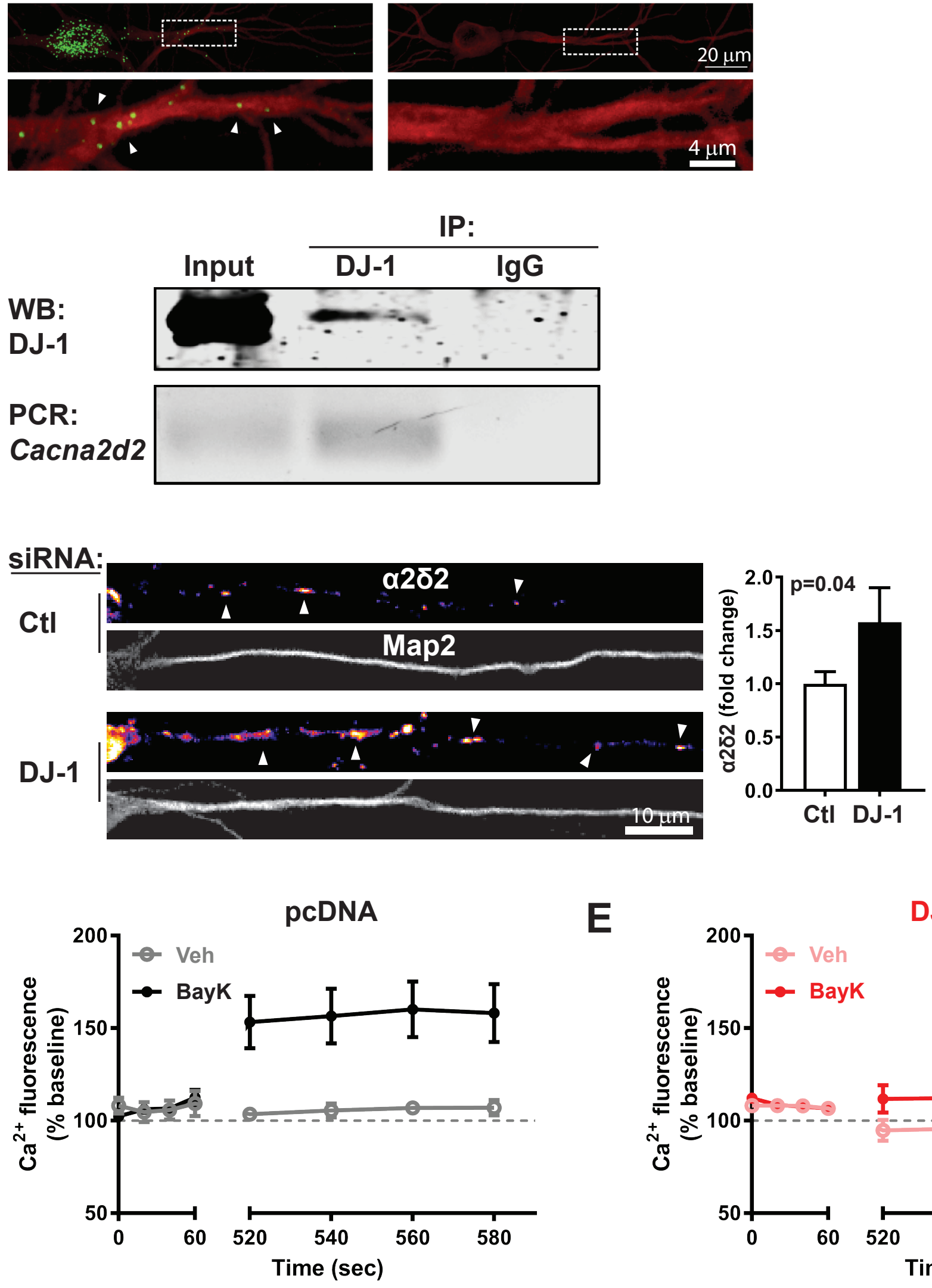

E
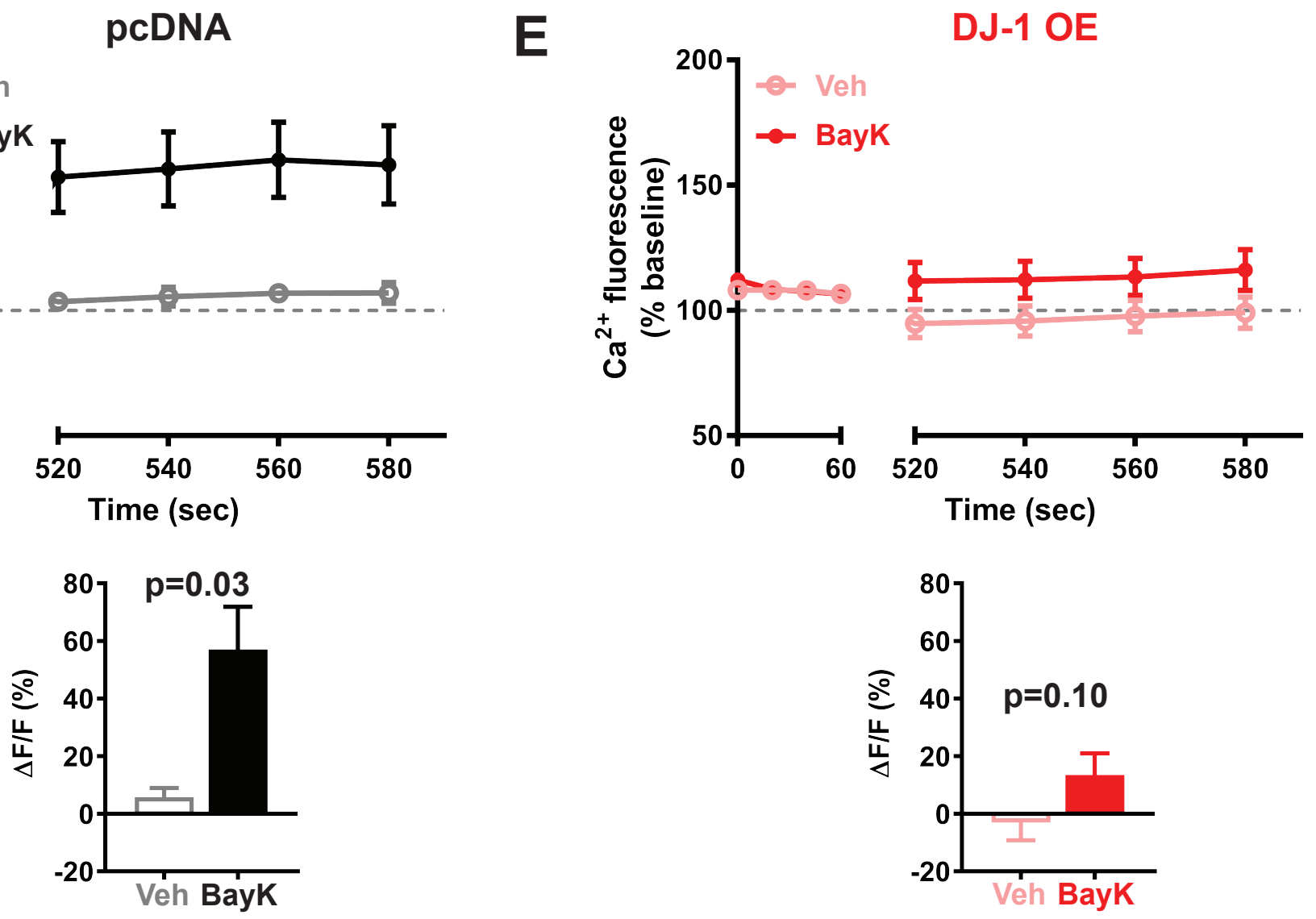


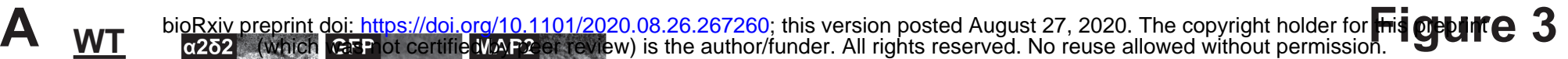
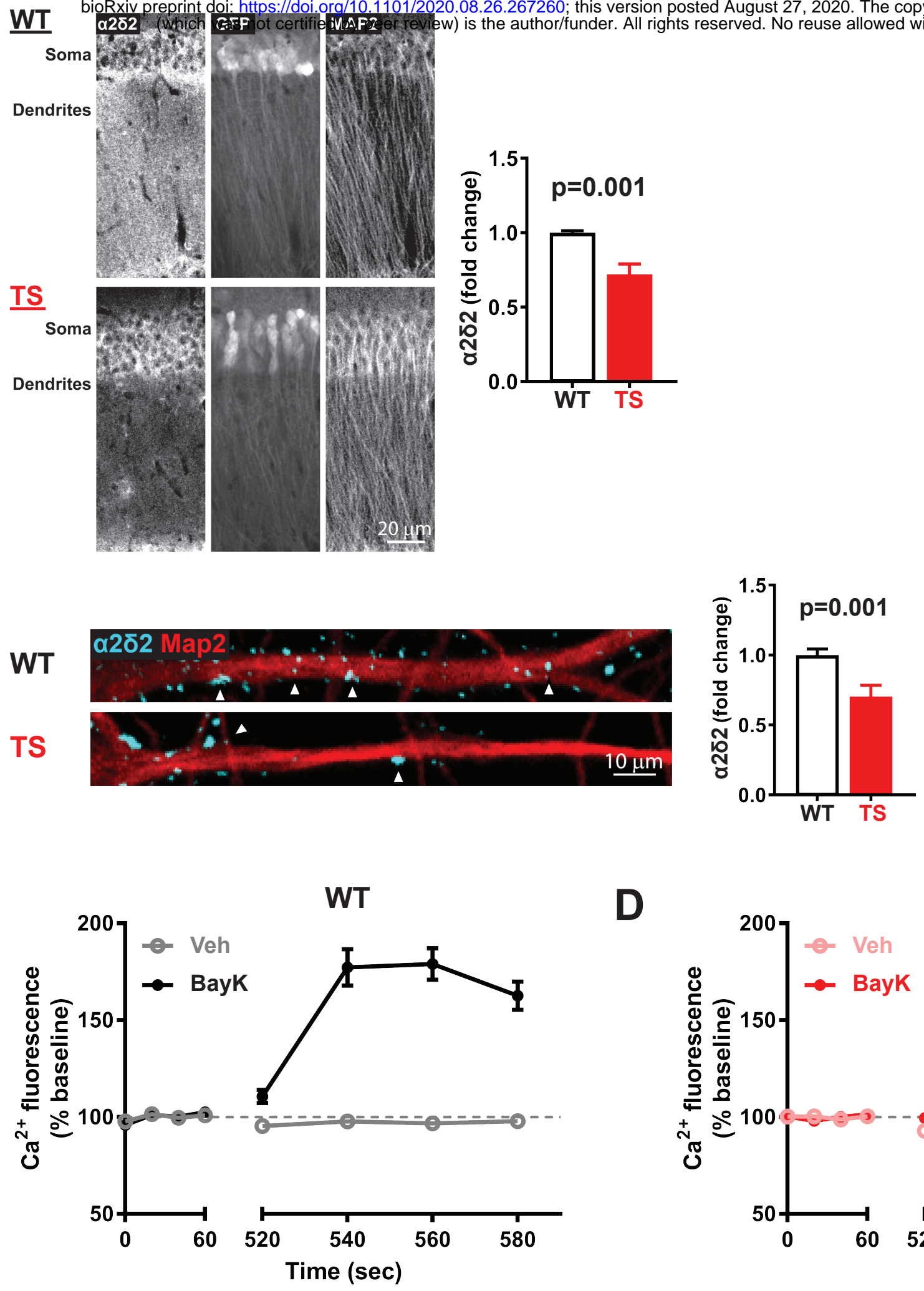

D
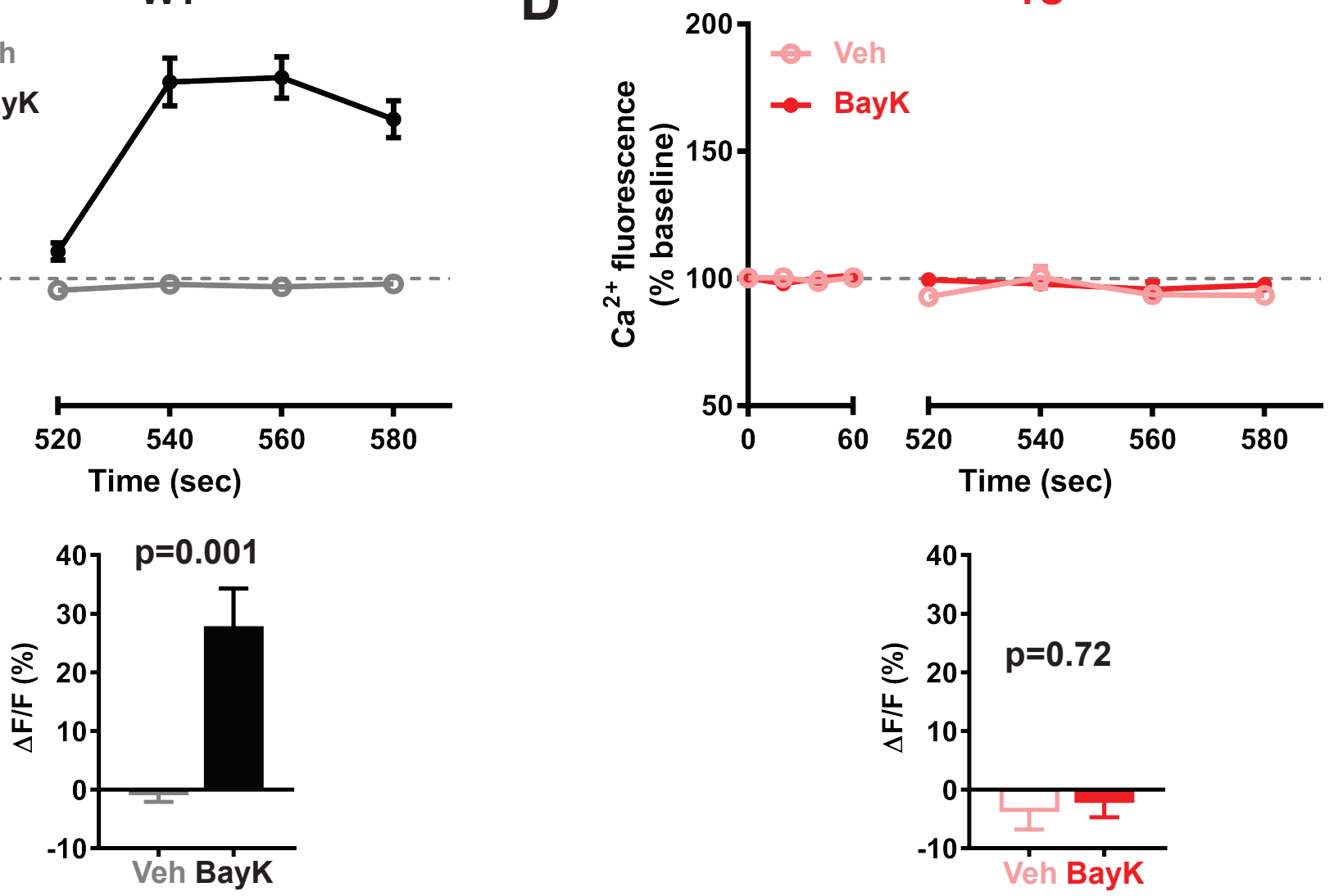
APP/

PS1
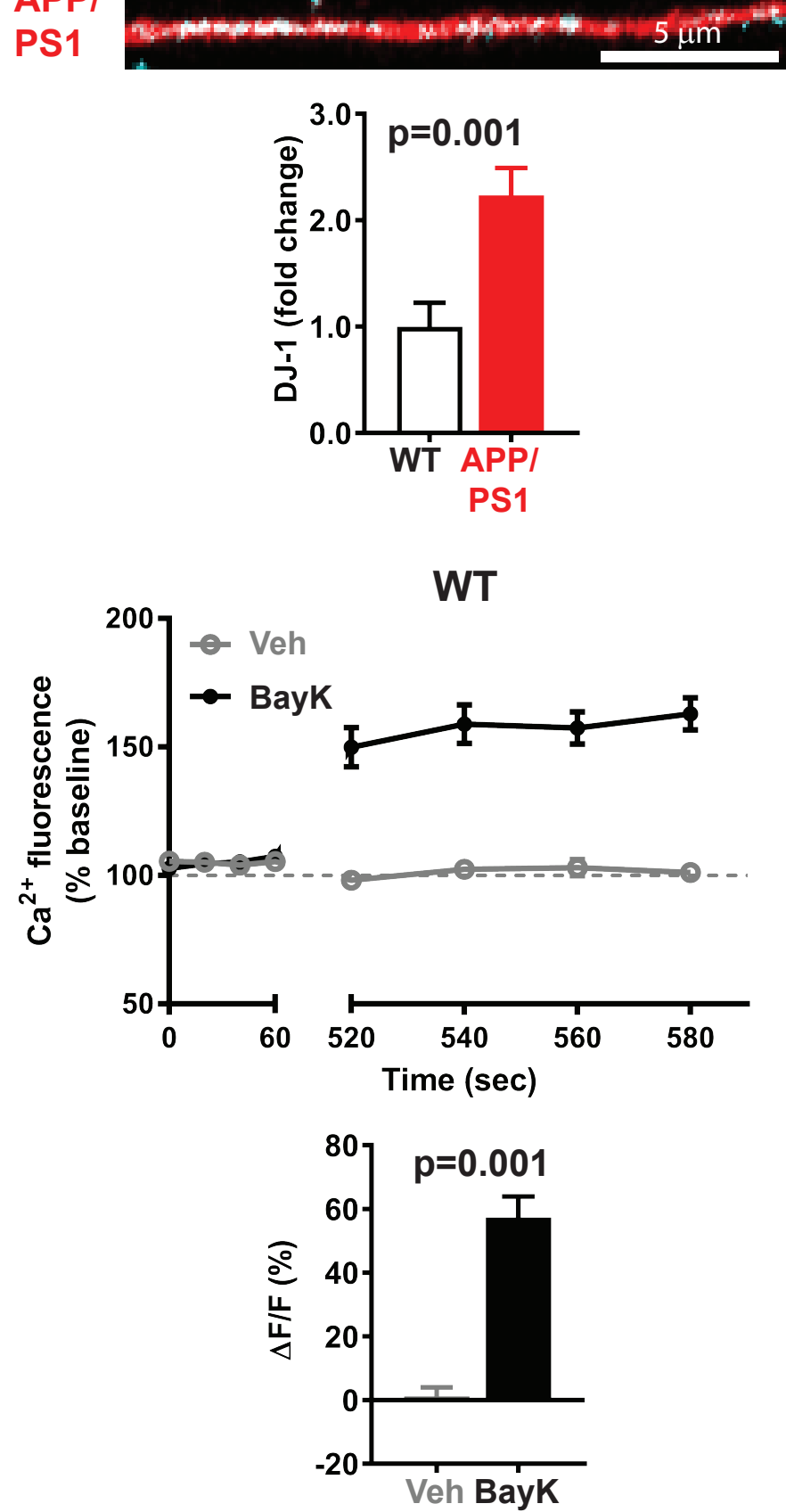

D
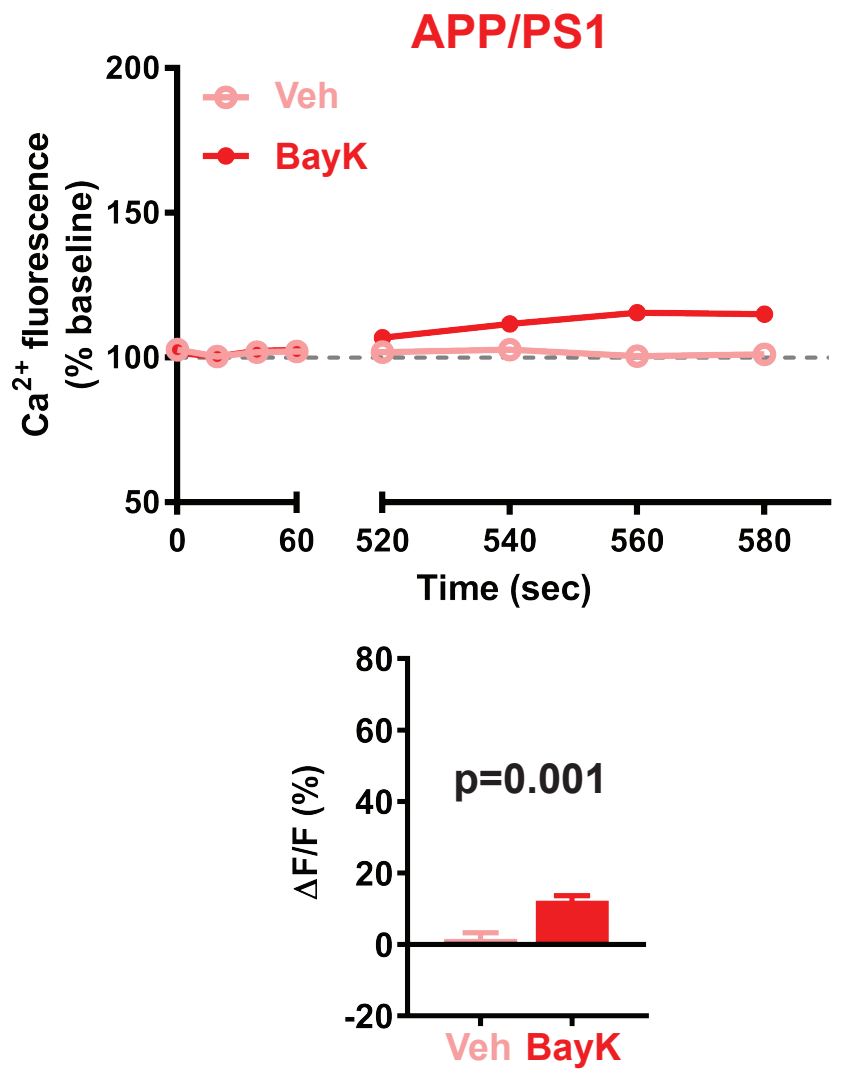

E

Ctl AD F $\quad$ Ctl AD $\quad$ G

DJ-1

Tubulin

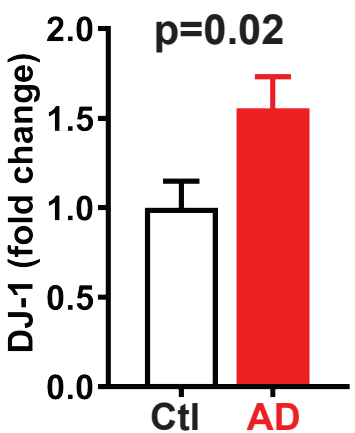

APP/

PS1

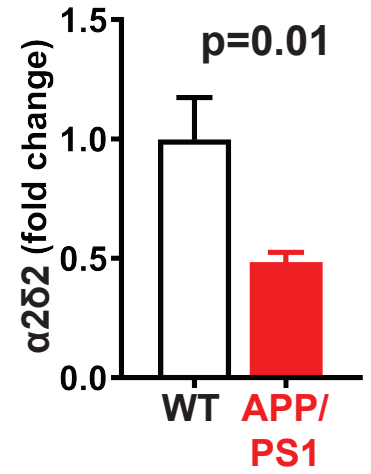

APP/PS1
pS6

Tubulin
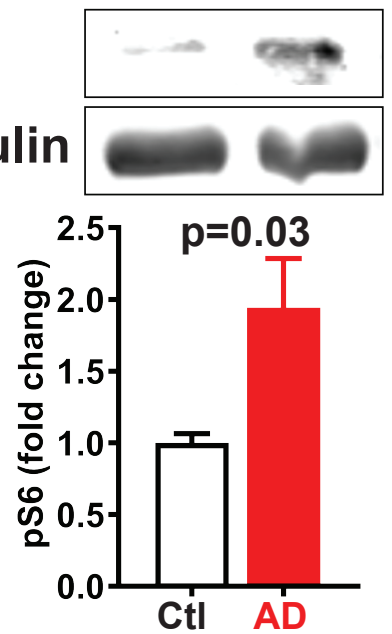
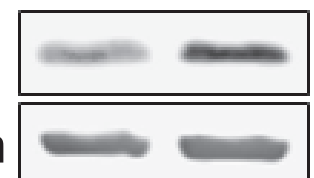

$\alpha 2 \delta 2$

Tubulin

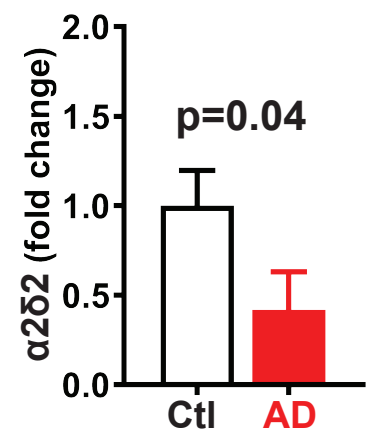

H

Ctl AD

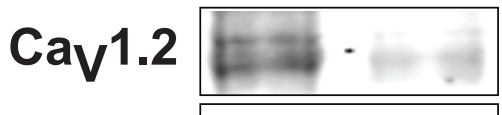

Tubulin

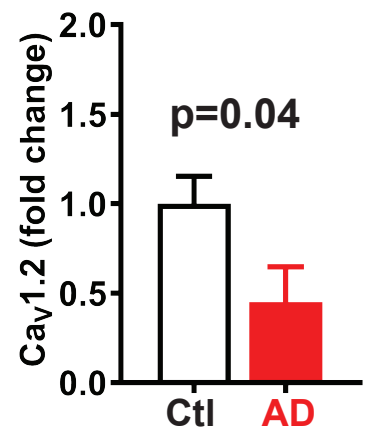

Figure 4 